\title{
MARTINGALE CONVERGENCE VIA THE SQUARE FUNCTION
}

\author{
LOUIS H. Y. CHEN ${ }^{1}$
}

\begin{abstract}
By exploiting the natural setting of a convergence theorem of Burkholder, a direct and elementary proof of the theorem is given. This proof is also new for the martingale convergence theorem and the martingale transform convergence theorem which are corollaries to the above-mentioned convergence theorem.
\end{abstract}

1. Introduction. An important generalization of Doob's martingale convergence theorem is Burkholder's [4] martingale transform convergence theorem. In [4], Burkholder also proves the following result:

TheOREM 1. Let $(\Omega, \mathscr{F}, P)$ be a probability space and $\mathscr{F}_{1} \subset \mathscr{F}_{2} \subset \ldots$ a nondecreasing sequence of sub-o-algebras of $\mathscr{F}$. Let $f=\left(f_{1}, f_{2}, \ldots\right)$ and $g=\left(g_{1}, g_{2}, \ldots\right)$ be two martingales relative to $\mathscr{F}_{1} \subset \mathscr{F}_{2} \subset \ldots$ Suppose $\|f\|_{1}<\infty$ and $S_{n}(g)<$ $S_{n}(f)$ for $n=1,2, \ldots$ Then $g$ converges a.s.

Here and throughout the rest of this paper, we adopt the following notation: for a martingale $f=\left(f_{1}, f_{2}, \ldots\right)$ relative to $\mathscr{F}_{1} \subset \mathscr{F}_{2} \subset \ldots$ and with difference sequence $d=\left(d_{1}, d_{2}, \ldots\right),\|f\|_{p}=\sup _{n}\left(E\left|f_{n}\right|^{p}\right)^{1 / p}, S_{n}(f)=\left(\sum_{i=1}^{n} d_{i}^{2}\right)^{1 / 2}, S(f)=$ $\sup _{n} S_{n}(f), f^{*}=\sup _{n}\left|f_{n}\right|, d^{*}=\sup _{n}\left|d_{n}\right|, f^{\tau}=\left(f_{1}^{\tau}, f_{2}^{\tau}, \ldots\right)$ denotes $f$ stopped at $\tau$, $d^{\tau}=\left(d_{1}^{\tau}, d_{2}^{\tau}, \ldots\right)$ denotes the difference of $f^{\tau}$, and $E_{n}$ denotes the conditional expectation operator given $\mathscr{F}_{n}$ for $n \geqslant 1$ and $E_{0}=E$.

It is easy to see that Theorem 1 is a generalization of both the martingale convergence theorem and the martingale transform convergence theorem.

Different proofs of the martingale convergence theorem abound in the literature (see, for example, [2], [9], [10] and [11]). There are also several proofs of the martingale transform convergence theorem some of which are extensions of the proofs of the martingale convergence theorem (see, for example, [3], [4], [8, pp. 72-74] and [10]). However there does not seem to exist in the literature other proofs than Burkholder's original proof of Theorem 1. The latter uses the martingale transform convergence theorem. (It is possible to deduce Theorem 1 from the deep inequality $\left\|f^{*}\right\|_{1} \leqslant C\|S(f)\|_{1}$ of Davis [7] by using a stopping time and then applying the martingale convergence theorem. But this is not our point.)

Received by the editors September 23, 1980 and, in revised form, November 28, 1980.

1980 Mathematics Subject Classification. Primary 60G42.

Key words and phrases. Martingale convergence, square function, martingale transform.

${ }^{1}$ This paper will be presented as a short talk at an International Mathematical Conference to be held in Singapore from June 1 to June 13, 1981. The Conference is organized by the Singapore Mathematical Society in conjunction with the Department of Mathematics, National University of Singapore. It is sponsored by UNESCO, the International Mathematical Union, the Mathematical Society of Japan and the Southwest Asian Mathematical Society. 
Actually the setting of Theorem 1 is very natural. It enables one to link the convergence of a martingale to its square function and use the elementary fact that the square function of an $L_{1}$-bounded martingale is finite a.s. (see, for example, [1], [5, p. 21], and [6]; see also the remark at the end of this paper). Thus the purpose of this paper is to give a direct an elementary proof of Theorem 1 which is also a new proof of the martingale convergence theorem and the martingale transform convergence theorem.

2. Proof. Let $d=\left(d_{1}, d_{2}, \ldots\right)$ and $e=\left(e_{1}, e_{2}, \ldots\right)$ be the difference sequences of $f$ and $g$ respectively. Define $\tau=\inf \left\{n:\left|f_{n}\right|>\lambda\right.$ or $\left.S_{n}(f)>\lambda\right\}$ where $\lambda>0$. Then

$$
\begin{aligned}
\left|e_{n}^{\tau}\right| & \leqslant S_{n}\left(g^{\tau}\right)=S_{\tau \wedge n}(g) \leqslant S_{\tau \wedge n}(f) \\
& =S_{n}(f) I(\tau>n)+S_{\tau}(f) I(\tau \leqslant n) \\
& \leqslant \lambda+\left|d_{\tau}\right| I(\tau \leqslant n)
\end{aligned}
$$

where $I(A)$ denotes the indicator function of the set $A$. Therefore

$$
e^{\tau *}<\lambda+\left|d_{\tau}\right| I(\tau<\infty) \leqslant 2 \lambda+\left|f_{\tau}\right| I(\tau<\infty)
$$

and so

$$
\begin{aligned}
E e^{\tau *} & \leqslant 2 \lambda+E\left|f_{\tau}\right| I(\tau<\infty) \\
& =2 \lambda+\sup _{n} E\left|f_{\tau \wedge n}\right| I(\tau<\infty) \\
& \leqslant 2 \lambda+\sup _{n} E\left|f_{\tau \wedge n}\right| \leqslant 2 \lambda+\|f\|_{1}<\infty
\end{aligned}
$$

where the inequality $\sup _{n} E\left|f_{\tau \wedge n}\right| \leqslant\|f\|_{1}$ follows from Doob's optional sampling theorem.

Now it is not difficult to see that

$$
E \sum_{n=1}^{\infty} \frac{e_{n}^{\tau 2}}{U_{n}^{2}} \leqslant E \int_{0}^{\infty} \frac{d x}{(1+x)^{2}}=1
$$

where $U_{n}=1+S_{n}^{2}\left(g^{\tau}\right)$. So if we let

$$
\xi_{n}=U_{n}^{-1} e_{n}^{\tau}-E_{n-1} U_{n}^{-1} e_{n}^{\tau}
$$

and define the martingale $h=\left(h_{1}, h_{2}, \ldots\right)$ relative to $\mathscr{F}_{1} \subset \mathscr{F}_{2} \subset \ldots$ by $h_{n}=$ $\sum_{i=1}^{n} \xi_{i}$, it will follow that $h$ is $L_{2}$-bounded. Indeed,

$$
\begin{aligned}
\|h\|_{2}^{2} & =\sup _{n} E h_{n}^{2}=\sum_{n=1}^{\infty} E \xi_{n}^{2} \\
& =\sum_{n=1}^{\infty} E E_{n-1} \xi_{n}^{2} \leqslant \sum_{n=1}^{\infty} E E_{n-1} U_{n}^{-2} e_{n}^{\tau 2} \\
& =E \sum_{n=1}^{\infty} U_{n}^{-2} e_{n}^{\tau 2}<\infty .
\end{aligned}
$$

Hence $h$ converges a.s. To emphasize the elementary character of this proof, we note that the a.s. convergence of an $L_{2}$-bounded martingale is a trivial generalization of a classical theorem concerning the a.s. convergence of sums of independent 
random variables. Now

$$
\begin{aligned}
E \sum_{n=2}^{\infty}\left|E_{n-1} U_{n}^{-1} e_{n}^{\tau}\right| & =E \sum_{n=2}^{\infty}\left|E_{n-1} U_{n}^{-1} e_{n}^{\tau}-E_{n-1} U_{n-1}^{-1} e_{n}^{\tau}\right| \\
& \leqslant E \sum_{n=2}^{\infty} E_{n-1} e^{\tau *}\left(U_{n-1}^{-1}-U_{n}^{-1}\right)<E e^{\tau *}<\infty
\end{aligned}
$$

and so $\sum_{n=1}^{\infty} E_{n-1} U_{n}^{-1} e_{n}^{\tau}$ converges absolutely a.s. It follows that $\sum_{n=1}^{\infty} U_{n}^{-1} e_{n}^{\tau}$ converges a.s. and in particular a.s. on $\{\tau=\infty\}=\left\{S(f)<\lambda, f^{*}<\lambda\right\}$. But on $\{\tau=\infty\}, e_{n}^{\tau}=e_{n}$ and $S_{n}\left(g^{\tau}\right)=S_{n}(g)$. So we have $\sum_{n=1}^{\infty}\left[1+S_{n}^{2}(g)\right]^{-1} e_{n}$ converges a.s. on $\left\{S(f) \leqslant \lambda, f^{*} \leqslant \lambda\right\}$ and hence on

$$
\left\{S(f)<\infty, f^{*}<\infty\right\}=\bigcup_{\lambda>0}\left\{S(f)<\lambda, f^{*}<\lambda\right\} .
$$

Now $\{S(g)<\infty\} \supset\{S(f)<\infty\}$ and it is easy to check that if $\sum_{n=1}^{\infty} a_{n}$ is a convergent series of real numbers and $\left\{b_{n}\right\}$ is a monotone sequence of real numbers with finite limit then $\sum_{n=1}^{\infty} a_{n} b_{n}$ converges. Using these we have $g$ converges a.s. on $\left\{S(f)<\infty, f^{*}<\infty\right\}$. But by Doob's maximal inequality $\|f\|_{1}<$ $\infty$ implies $f^{*}<\infty$ a.s. and by Austin's result [1] $\|f\|_{1}<\infty$ implies $S(f)<\infty$ a.s. (see also [5, p. 21] and [6]). It follows that $g$ converges a.s. This proves the theorem.

REMARK. In [1], although the martingale convergence theorem is quoted in the last step of the proof, it is Doob's maximal inequality that is really needed. Similarly, in [5], it is not necessary to use Lemma 2.1 to prove Theorem 3.1 ; the inequality (2.2) is sufficient.

\section{REFERENCES}

1. D. G. Austin, A sample function property of martingales, Ann. Math. Statist. 37 (1966), 1396-1397. MR 33 \#6687.

2. Luis Báez-Duarte, Another look at the martingale theorem, J. Math. Anal. Appl. 23 (1968), 551-557. MR 37 \# 4859.

3. 319-322. MR 45 \#9374.

4. D. L. Burkholder, Martingale transforms, Ann. Math. Statist. 37 (1966), 1494-1504. MR 34 \#8456.

5. \# 1944. , Distribution function inequalities for martingales, Ann. Probab. 1 (1973), 19-42. MR 51

6. Louis H. Y. Chen, A martingale inequality for the square and maximal functions, Ann. Probab. 7 (1979), 1051-1055.

7. Burgess Davis, On the integrability of the martingale square function, Israel J. Math. 8 (1970), 187-190. MR 42 \#3863.

8. Adriano M. Garsia, Martingale inequalities: seminar notes on recent progress, Benjamin, New York, 1973. MR 56 \#6844.

9. Richard Isaac, A proof of the martingale convergence theorem, Proc. Amer. Math. Soc. 16 (1965), 842-844. MR 31 \#4071.

10. Charles W. Lamb, A short proof of the martingale convergence theorem, Proc. Amer. Math. Soc. 38 (1973), 215-217. MR 48 \#3119.

11. Paul-André Meyer, Martingales and stochastic integrals. I, Springer-Verlag, Berlin and New York, 1972.

Department of Mathematics, National University of Singapore, Bukt Tmaah Road, SingaPORE 1025, RePUblic OF SingAPORE 